Ann. Sci. forest., 1974, 31 (2), 111-128.

\title{
BIOLOGIE ET DÉGATS D'HEXACOLUS GUYANENSIS SCHEDL. DANS LES PLANTATIONS D'ACAJOU ROUGE (SWIETENIA MACROPHYLLA KING., MÉLIACÉE) EN GUADELOUPE (COLEOPTERA : SCOLYTIDAE)
}

\section{GRUNER}

avec la collaboration technique de D. Marival

Station de Zoologie et de Lutte biologique, C.R.A.A.G., I.N.R.A., 97170 Petit Bourg (Guadeloupe)

\section{RÉSUMÉ}

La biologie d'un ravageur primaire de l'acajou rouge, Hexacolus guyanensis a été étudiée au laboratoire et dans la nature. Cette espèce est monogame, et quatre stades larvaires se développent en deux à trois semaines dans le phloème. Elle semble inféodée à quelques représentants de la famille des Meliacées.

L'évolution de la population de Scolytes a été suivie au cours du dépérissement de l'arbre, ainsi que la présence de la faune associée et de germes. La larve prédatrice du Cléride Madoniella pici est décrite.

L'étendue des dégâts depuis 1967 en Guadeloupe nous a fait conseiller la coupe suivie du brûlage des arbres infestés. L'efficacité de cette méthode a été vérifiée.

Deux foyers de cet insecte dans les peuplements de mahogany ont été suivis ainsi que l'évolution des populations du ravageur et de son complexe parasitaire. Le développement d'une epizootie à Metarrhizium anisopliae a permis d'expliquer l'extinction naturelle de l'un de ces foyers. couleur.

Le repérage des foyers par télédétection a montré l'intérêt des émulsions infra rouge fausse

Introduit du Honduras en Guadeloupe vers 1945, l'acajou rouge ou Mahogany (Swietenia macrophylla KING.) a pris une grande extension. Depuis 1949, 2400 hectares ont été plantés, principalement entre 1960 et 1964, et il en est prévu 10000 hectares d'ici 1980.

Parmi les revageurs les plus importants (LAMB, 1966), il faut signaler aux Antilles les attaques des jeunes plants par Hypsipyla grandella M. G. Pyralidae dont les larves minent les pousses terminales pendant la saison humide. Les arbres survivent mais avec des déformations longues à disparaître.

Depuis quelques années, un autre ravageur est apparu en Guadeloupe, le scolyte Hexacolus guyanensis ScHEDL. Décrit en Guyane Britannique et trouvé au Surinam égale- 
ment sur acajou, son introduction aux Antilles semble récente. Inconnu jusqu'à présent en Martinique, d'importantes pullulations sont constatées depuis 1967 dans les plantations de la Guadeloupe, au point d'inquiéter les autorités qui ont ralenti le rythme des nouvelles plantations et recherché d'autres essences.

La biologie de cet insecte étant inconnue, nous avons en premier lieu défini son cycle biologique avant de suivre l'évolution des populations au cours de la dégradation de l'arbre hôte ainsi que le développement de foyers d'attaque dans les plantations. L'action des facteurs naturels et des coupes sanitaires sur la population d'insectes a été examinée.

\section{I. - BIOLOGIE DU RAVAgEUR}

Des morceaux de tronc d'acajou rouge de 7 à $9 \mathrm{~cm}$ de diamètre et de $25 \mathrm{~cm}$ de longueur, indemnes de toute attaque par des insectes, sont placés au laboratoire dans une enceinte en présence de morceaux de tronc infestés par $H$. guyanensis. Des scolytes adultes migrent et forent des galeries dans les troncs sains. Par des contrôles réguliers, les orifices initiaux sont repérés et datés afin de connaître l'âge des galeries dont la dissection nous permet de suivre la formation et la succession des générations d'insectes.

\section{1. - Formation des galeries}

Les insectes migrant sur le tronc traversent l'écorce et forment dans le phloème une chambre d'accouplement. Il est difficile de connaître avec exactitude le sexe des insectes qui creusent cette première galerie, mais il semble que ce soit les femelles, comme chez Alni-

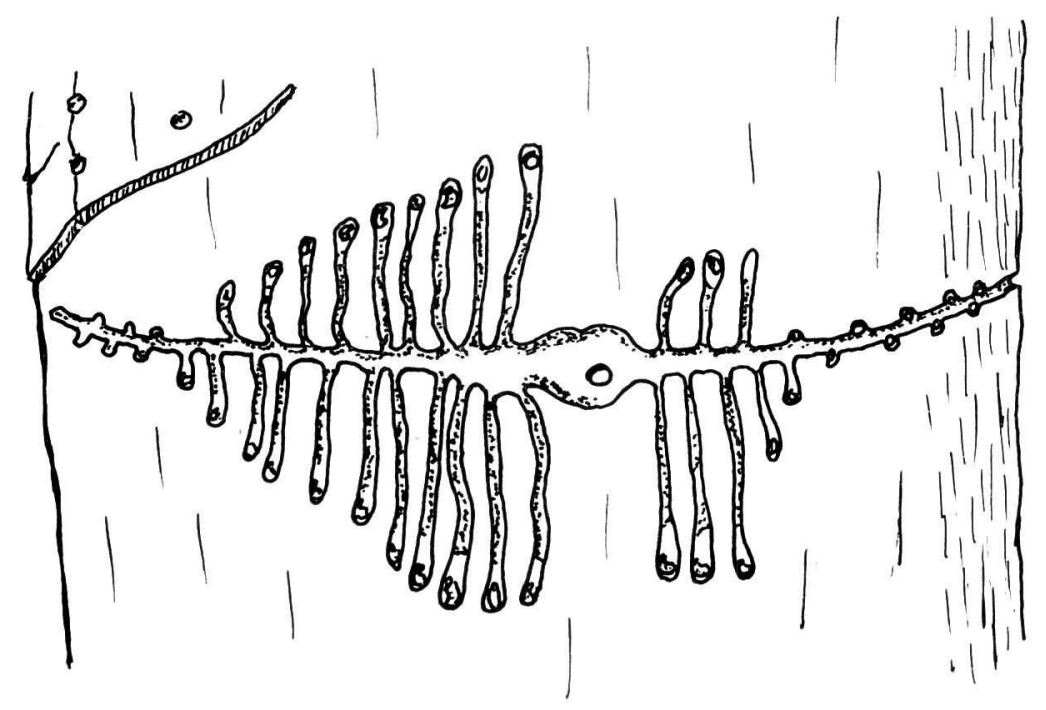

FIG. 1. - Galeries d'H. guyanensis dans le phloème du Mahogany du Honduras

FIG. 1. - Galeries of $\mathrm{H}$. guyanensis in the mahogany bast Honduras 
phagus aspericollis (Borden, 1969), et à l'inverse de Corthylus columbianus (Milne, 1968). Si une participation des mâles est possible, ce sont les femelles qui forent selon un plan horizontal, la galerie de ponte, sur une longueur de l'ordre de 10 à $12 \mathrm{~mm}$ de part et d'autre de l'orifice d'entrée. Cette forme de galerie de ponte transversale dite en accolade (BALACHOWSKY, 1963) rappelle celle de l'espèce monogame Blastophagus minor HART, sur Pin sylvestre (Pesson, Chararas, 1969). Les œufs sont déposés de chaque côté de cette galerie, au fur et à mesure de sa formation. Les galeries larvaires, rectilignes et longitudinales dans le phloème, s'alternent le long de la galerie maternelle, les plus longues étant les plus proches de la chambre d'accouplement (fig. 1). Les larves ont un comportement analogue à celui décrit par BoRden (1969) chez Alniphagus aspericollis (Le Comte), au fur et à mesure de leur progression elles tassent derrière elles les matières fécales et bouchent ainsi la galerie, ce qui limite l'introduction de prédateurs ou de parasites. En fin d'évolution, elles aménagent une loge nymphale. Le jeune imago soit creusera un trou de sortie vers l'extérieur et migrera, soit reviendra vers la galerie maternelle. Certains s'y accouplent et l'allongent pour y déposer à nouveau des œufs, bouclant le cycle à l'intérieur du tronc.

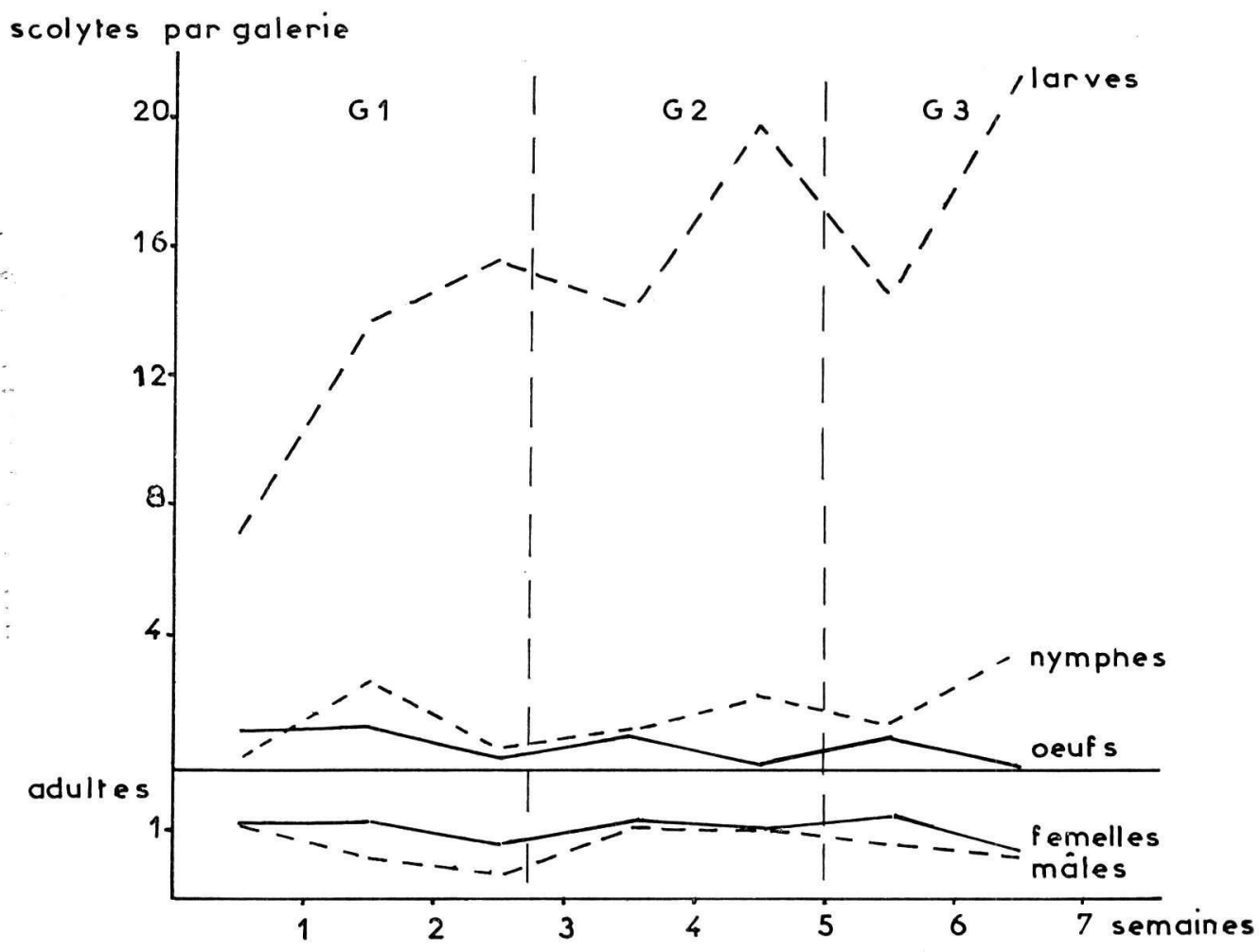

FIG. 2. - Évolution du nombre de Scolytes suivant l'âge des galeries FIG. 2. - Evolution of the number of bark beetles with the age of the galeries 


\section{2. - Développement des insectes dans les galeries}

La figure 2 illustre le résultat des dissections de galeries et présente le nombre d'adultes et leur descendance en fonction de l'âge de l'orifice initial, l'unité étant l'ensemble des galeries maternelles et larvaires aboutissant à cet orifice. Les galeries abandonnées en début de formation ne sont pas prises en considération.

Au cours de la $1^{\text {re }}$ semaine, les adultes creusent la galerie de ponte et les femelles commencent à déposer les œufs. Dans $10 \%$ des galeries il y a plus d'un couple dans la loge d'accouplement.

La $2^{\text {e }}$ semaine, les mâles quittent les galeries pour aller en initier une autre ou pour migrer vers un autre tronc, tandis que œufs et larves sont en grand nombre. La présence de nymphes indique que la durée d'évolution larvaire peut-être inférieure à 2 semaines.
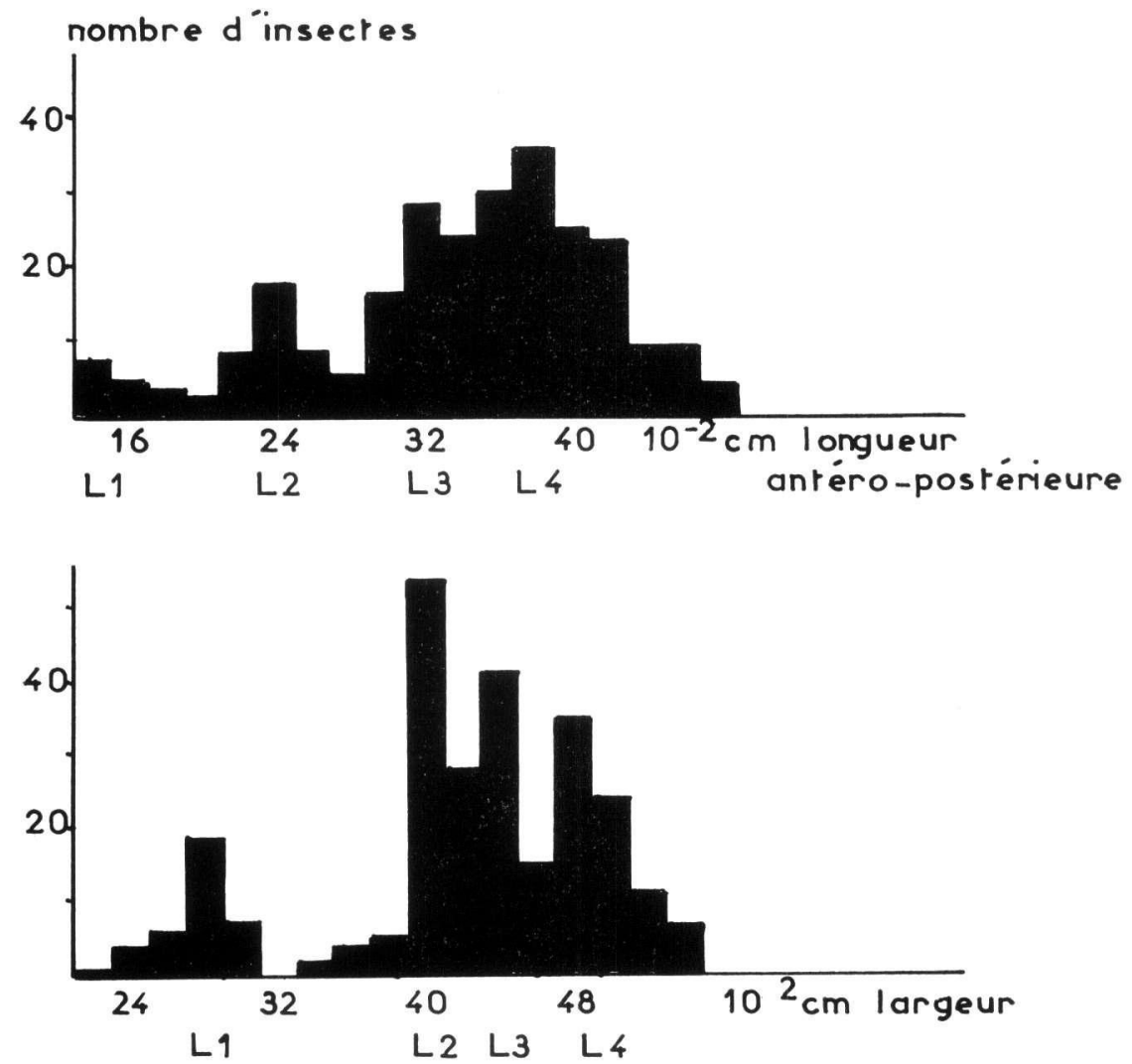

FIG. 3. - Distribution de la longueur anétro-postérieure (en haut) et de la plus grande largeur des capsules céphaliques d'H. guyanensis

FIG. 3. - Distribution of the fore to backside length (up) and of the larger width of the head of $\mathrm{H}$. guyanensis larvae 
La $3^{\text {e }}$ semaine, peu d'adultes sont dans les galeries, et la ponte est terminée, chaque femelle ayant déposé une moyenne de 15 œufs en 2 à 3 semaines. De jeunes adultes ont déjà éclos, de sorte que la durée de développement de l'œuf à l'adulte est comprise entre 2 et 3 semaines.

La $4^{\text {e }}$ semaine, la nette augmentation du nombre d'adultes, même dans des morceaux de troncs isolés d'infestations extérieures, indique que les jeunes adultes dès leur naissance peuvent infester le même tronc. Cette période de ponte correspond au début de la $2^{\mathrm{e}}$ génération ( $\mathrm{G}$ 2).

$5^{\mathrm{e}}$ semaine : la ponte de cette $2^{\mathrm{e}}$ génération est terminée et le nombre de larves et de nymphes correspondant aux générations $G 1$ et $\mathrm{G} 2$ est élevé.

$6^{\text {e }}$ semaine : nouvel apport de femelles et d'œufs ( $3^{\mathrm{e}}$ génération).

$7^{\text {e }}$ semaine : diminution du nombre d'adultes comme 2 semaines auparavant, la période de ponte G 3 est terminée, G 2 et G 3 poursuivent leur développement. Cette forte densité larvaire est due au fait que les générations se chevauchent dans les mêmes galeries, c'est-àdire que de jeunes adultes restent dans la galerie maternelle sans quitter le tronc.

Au delà de ce délai, le dessèchement progressif des morceaux de tronc limite le développement des insectes, et les jeunes adultes migrent à la recherche d'un nouvel hôte.

\section{3. - Nombre de stades larvaires}

Les mesures de la plus grande largeur et de la longueur antéropostérieure des capsules céphaliques de 270 larves d'Hexacolus guyanensis d'âges et de dimensions variées font apparaître 4 stades larvaires (Figure 3), ayant des largeurs de capsule céphalique respectivement de $0,28-0,40-0,44$ et $0,50 \mathrm{~mm}$. L'analyse statistique de ces mesures confirme ces résultats, les différences observées étant significatives au seuil de $1 \%$. Ce nombre de 4 stades larvaires précédant la nymphose correspond à celui de la plupart des Scolytes, bien que Ips cembrae en ait 3 (BAlogun, 1970), ainsi que Alniphagus aspericollis (BoRden, 1969).

\section{4. - Gamme d'hôtes}

Les observations d'arbres attaqués dans la nature ont été complétées par des expériences d'infestation artificielle au laboratoire en mettant en contact des morceaux de troncs de diverses essences avec de l'acajou infesté par Hexacolus guyanensis, ou bien en disposant sur le tronc d'arbres sur pied un manchon contenant du mahogany infesté.

Le Mahogani petites feuilles (Swietenia mahagoni Jacq.) et l'acajou pays (Cedrela odorata L.) se sont révélés sensibles aux attaques de l'insecte. Bien que leur présence dans les plantations soit exceptionnelle, nous avons observé quelque cas d'attaque en milieu naturel lorsqu'un représentant de ces espèces se trouve entouré de Mahogani grandes feuilles attaqués par le Scolyte. Le fait que ces essences soient endémiques tend à prouver qu'Hexacolus guyanensis a été introduit en Guadeloupe, n'ayant jamais été récolté auparavant dans ce département malgré de nombreuses prospections faunistiques. Il semble néanmoins inféodé à quelques essences de la famille des Méliacées. 
Le tableau 1 résume l'ensemble des observations effectuées :

TABLEAU 1

Sensibilité de différentes essences vis-à-vis d'Hexacolus guyanensis

Sensibility of different varieties to Hexacolus guyanensis infestations

\begin{tabular}{|c|c|c|c|}
\hline FAMILLE & ESSENCE & NON COMMUN & $\begin{array}{l}\text { SENSIBILITÉ } \\
\text { AU SCOLYTE }\end{array}$ \\
\hline Meliacées & $\begin{array}{l}\text { Swietenia macrophilla King. } \\
\text { Swietenia mahagoni Jacq. } \\
\text { Melia azedarach L. } \\
\text { Cedrela odorata } \mathrm{L} . \\
\text { Anthocephalus cadamba } \\
\text { Guarea trichiloides L. } \\
\text { Hibiscus elatus W. } \\
\text { (H. tiliaceus } \mathrm{L} .)\end{array}$ & $\begin{array}{l}\text { Mahogany du Honduras } \\
\text { Mahogani petites feuilles } \\
\text { Lila pays } \\
\text { Acajou amer, acajou pays, } \\
\text { Acajou senti. } \\
\text { Cadam. } \\
\text { Neflier des bois, } \\
\text { bois pistolet. } \\
\text { Bois de liège } \\
\text { Mahot gombo. }\end{array}$ & $\begin{array}{l}++t+ \\
++t+ \\
\end{array}$ \\
\hline
\end{tabular}

\section{II. - DÉVElopPEMENT DE L'ATTAQUE SUR L'ACAJOU ROUGE}

\section{1. - Manifestations sur l'arbre (Fig. 4 à 6)}

Hexacolus guyanensis est un ravageur primaire de feuillus, ce qui est relativement peu commun chez les représentants de la tribu des Ipini (Chararas, 1970).

Il attaque des arbres apparement sains, ayant au moins 9 à 10 ans. Le diamètre du tronc n'intervient pas car nous avons constaté des attaques sur des arbres particulièrement bien développés dont le tronc dépassait $30 \mathrm{~cm}$ de diamètre, aussi bien que sur des sujets chétifs, sous ombrage, ayant un tronc de 6 à $8 \mathrm{~cm}$.

Les premiers insectes migrants percent leurs galeries à une hauteur de 2 à 3 mètres, et des goutelettes de sève s'écoulent par les orifices d'entrée. Cette exsudation caractéristique de l'attaque devient vite importante, et s'étale vers le bas comme vers le haut, jusqu'à la naissance des premières branches. Dans le mois qui suit, les feuilles jaunissent, puis sèchent et tombent. Deux mois après la perte de sève, l'arbre est mort, sec, et est envahi par les termites. Leur présence confirme la mort du végétal, la sève du mahogany étant connue comme répulsive pour les termites (ASENJO et al. 1958).

\section{2. - Population de Scolytes et dégâts}

Au lieu dit Desbonnes, nous avons suivi une importante infestation en 1970 en effectuant des prélèvements de morceaux d'écorce sur 10 arbres en début d'attaque, toutes les 3 semaines. 


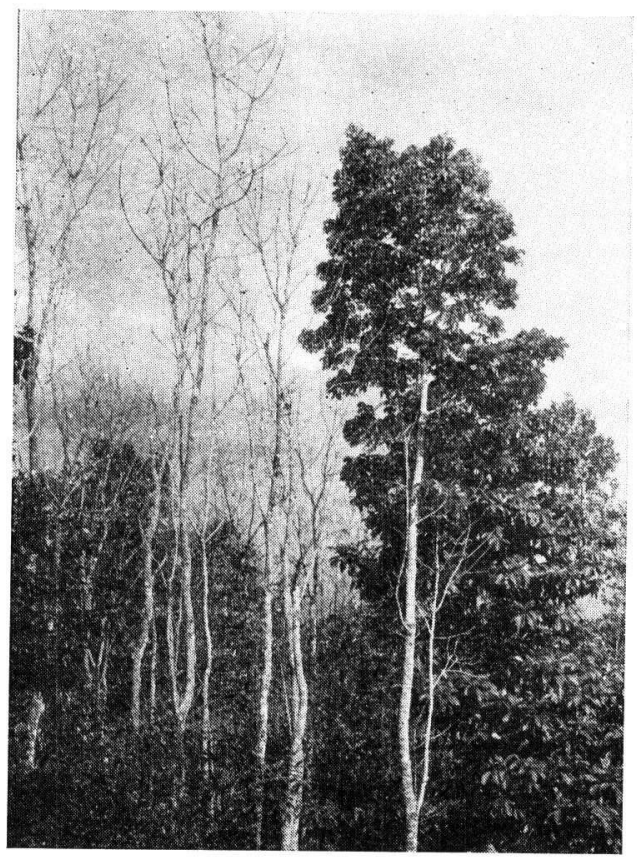

FIG. 4. - Acajous rouges sains et attaqués par H. guyanensis

FIG. 4. - Normal and infested by H. guyanensis mahogany trees

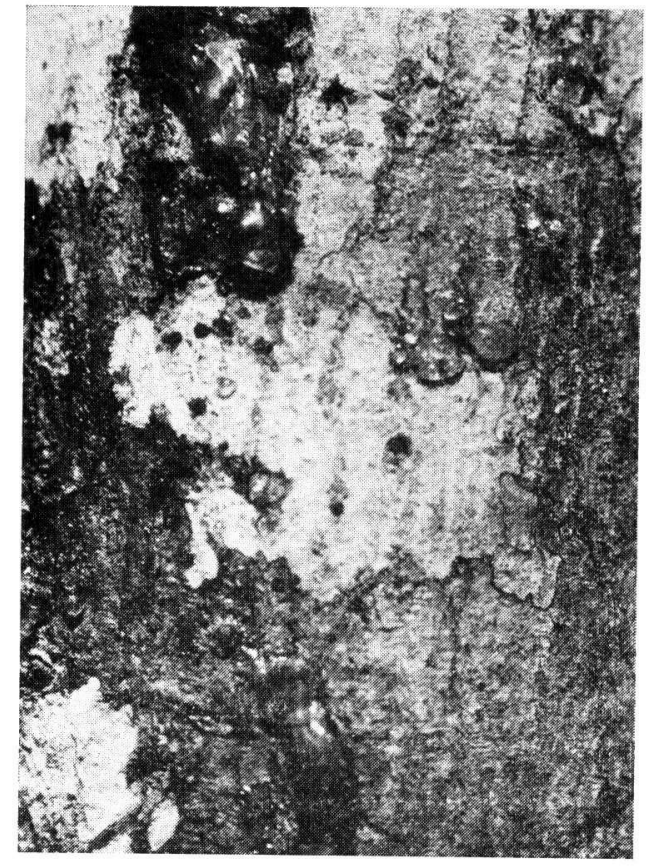

FIG. 5. - Orifices des galeries d'H. guyanensis

FIG. 5. - Apertures of the $\mathrm{H}$. guyanensis galeries

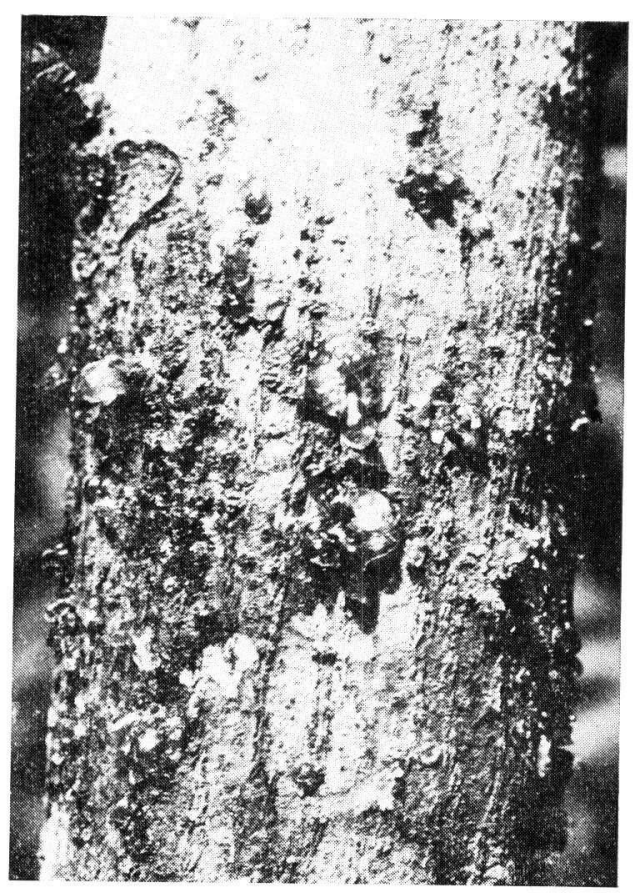

FIG. 6. - Importante coulée de sève au cours d'une forte attaque du Mahogany par le Scolyte

FIG. 6. - An important sap outflow during a hard infestation of a mahogany tree by the bark beetle 
Les facteurs hauteur sur le tronc (à hauteur d'homme et près du sol) et orientation par rapport au sens du vent dominant furent pris en considération au moment des prélèvements. Au laboratoire, 2 morceaux de $10 \mathrm{~cm}^{2}$ par échantillon furent disséqués avec notation du nombre d'orifices d'entrée ou de sortie, du nombre d'adultes et de larves.

Nous avons ainsi constaté que :

- Le nombre d'orifices d'entrée des galeries est plus important à 2 mètres qu'à $50 \mathrm{~cm}$, au début de l'infestation qui s'étend vers le bas. Nous n'avons pas effectué des prélèvements complémentaires à $4 \mathrm{~m}$ du sol en raison des difficultés techniques que cela soulevait.

- Le côté au vent du tronc reçoit directement la pluie, et est recouvert de lichens. A la première phase de l'attaque le nombre d'orifices est très nettement supérieur sur le côté sous le vent, alors que dès qu'elle se propage, ce facteur orientation n'intervient plus, et la répartition des orifices est uniforme tout autour du tronc.

- La figure 7 résume l'évolution d'une attaque en prenant comme critère le nombre total d'insectes et le nombre d'adultes d'Hexacolus, ainsi que la densité des orifices d'entrée ou de sortie des galeries en fonction des diverses phases de dégradation de l'arbre. Il apparaît que le début d'écoulement de sève attire de très nombreux insectes et provoque une infestation massive de l'arbre : le nombre d'orifices d'entrée s'accroît rapidement, ainsi que les densités de scolytes, le maximum se situant à la période de pleine suda $₫$ ion, avec 200 scolytes par $100 \mathrm{~cm}^{2}, 3$ semaines plus tard. Puis les insectes quittent les galeries alors que la première génération de larves donne des adultes dont une partie forent de nouveaux orifices

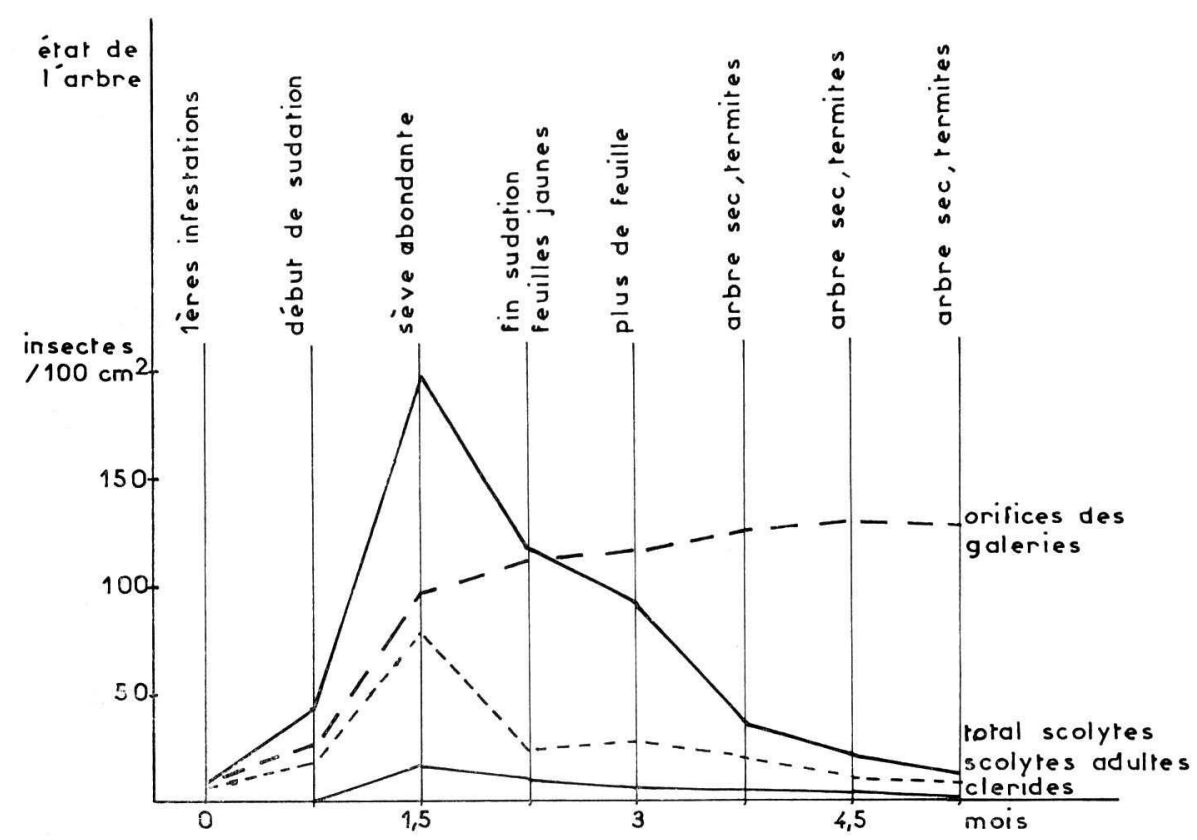

Fig. 7. - Évolution de l'arbre, de la population d'H. guyanensis et de son Cléride prédateur FIG. 7. - Evolution of the tree, of the $\mathrm{H}$. guyanensis population and of its clerid predator 
de sortie, ce qui augmente la densité globale des orifices. Une autre partie reste dans le phloème pour y pondre, formant d'autres générations jusqu'à ce que celui-ci soit totalement consommé. Les insectes quittent alors le tronc pour essaimer vers d'autres arbres. Entre le moment de l'infestation massive et la perte des feuilles, il ne s'est guère écoulé plus d'un mois et demi, l'arbre ne tardant pas à sécher et à être envahi par les termites.

Ce mécanisme de colonisation de l'arbre sous forme d'une première attaque et début d'exsudation, puis attraction des insectes et infestation massive entraînant une forte exsudation et enfin essaimage des générations suivantes a déjà été montré pour de nombreux Scolytes, tels par exemple divers Dendroctonus sp. (BAKKE 1973, RENWICK et ViTE 1969, 1970), et Scolytus ventralis (Berryman et Ashraf 1970).

\section{3. - Sorties des adultes}

Nous avons complété notre étude en mettant dans des éclosoirs, des rondins d'un tronc ayant subi depuis peu une forte infestation, afin de suivre les sorties des adultes de Hexacolus guyanensis. Par cette méthode, nous récoltons les insectes qui quittent le tronc.

La comparaison des sorties à différentes hauteurs du tronc fait apparaître qu'elles sont simultanées, les maxima et minima coïncidant pour les 3 hauteurs considérées : base du tronc, 3 à 5 mètres et proximité des premières branches. Il semblerait donc que des facteurs externes influencent ces sorties. Les dates de maxima correspondent à des jours où le temps est couvert avec une forte humidité relative et surtout une faible insolation. Cependant l'influence de tels facteurs dans les éclosoirs est pratiquement nulle. WATSON (1970) avait expliqué par l'existence d'un rythme circadien, la coïncidence des sorties de Dendroctonus ponderosae des morceaux de tronc placés au laboratoire dans des conditions de température et de lumière stables avec celles des troncs de la nature.

La courbe de sortie des adultes (Fig. 8) présente plusieurs maxima correspondant au chevauchement des générations $\mathrm{G} 1, \mathrm{G} 2, \mathrm{G} 3$ et $\mathrm{g} 1$ et $\mathrm{g} 2$. Deux à trois semaines séparent

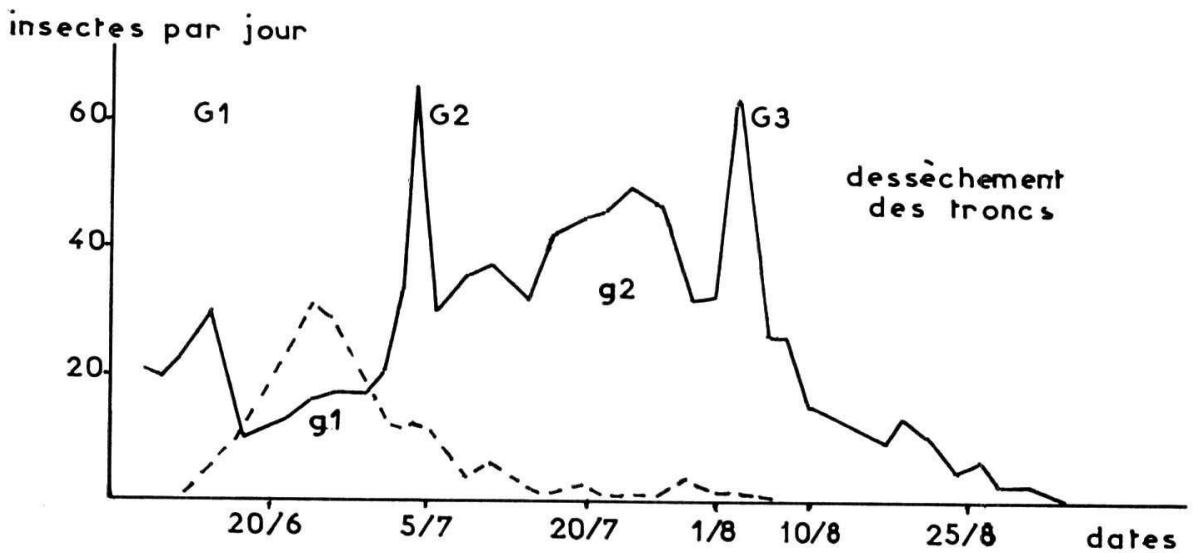

FIG. 8. - Sorties d'H. guyanensis (trait plein) et du Cléride (trait pointillé) en éclosoir FIG. 8. - Departures of $\mathrm{H}$. guyanensis (full line) and of the Clerid beetle (dotted line) in an hatching box 
les générations successives ce qui confirme les observations biologiques sur la durée du cycle, et le fait qu'une partie des jeunes imagos ne migre pas mais subsiste dans le tronc pour s'accoupler et pondre.

\section{4. - Faune et maladies associées}

\section{a. Scolytes *}

Diverses espèces se rencontrent également sur l'acajou rouge, la plupart pénètrent dans l'aubier, mais leur présence est occasionnelle. Il s'agit de :

- Pterocyclon praeustum Eggers.

- Neodryocoetes guadeloupensis Schedl. (= Neopithyophthorus insularis Eggers)

- Xyleborus aspericauda Egg.

- Xyleborus cavipennis Eich.

- Xyleborus ferrugineus $\mathrm{F}$.

- Stephanoderes gracilis Egg.

\section{b. Maladies}

La muscardine verte à Metarrhizium anisopliae Sorokin. entraîne une mortalité importante tant larvaire qu'adulte des Scolytes dans les galeries. Cette souche appartient au type minor avec des spores particulièrement courtes et ovoïdes, de 3,5 $\times 2,3$ microns. Des saprophytes du type Penicillium sp et Phaeo scopulariopsis sont également observés dans les galeries. La présence de tels germes a déjà été mise en évidence chez Dendroctonus frontalis Zimm. (Moore 1970).

\section{c. Prédateurs}

Dans les galeries, des larves d'un Ostomidae indéterminé consomment des larves d'Hexacolus guyanensis, mais sa présence est rare. Il en est de même pour un petit Staphylinidae. Par contre un Clèride se rencontre couramment dans les pullulations de scolytes : il s'agit de l'espèce décrite de Guadeloupe par LePESME (dans Fleutiaux et al. 1947) comme étant Madoniella pici (Cleridae : Corynetinae).

Cette espèce étant peu connue, nous donnons la description de la larve inconnue jusqu'à présent : corps très allongé, déprimé, membraneux à soies fines peu nombreuses, pâles. Capsule céphalique subrectangulaire, d'un quart plus longue que large, convexe dorsalement, sans sculpture apparente, bien chitinisée, ocre-brunâtre. Prothorax sub-rectangulaire, légèrement plus long que large, un peu plus grand que le meso et le matathorax, à plaque dorsale bien chitinisée, ocre clair. Pattes légèrement chitinisées au dernier stade. Abdomen membraneux, sans partie chitinisée sauf sur l'article 9 au $1^{\text {er }}$ stade. Au $3^{\mathrm{e}}$ stade présence de 4 épines, tandis qu'au $4^{\mathrm{e}}$ stade 2 plaques brunes ornent la partie dorsale. Scolytes.

* Je remercie M. Menier du Muséum d'Histoire Naturelle de Paris pour la détermination de ces 
Ces modifications dans la morphologie du dernier segment abdominal de la larve nous ont permis de distinguer 4 stades comme ReID (1957) l'avait observé chez Enoclerus sphegeus $\mathrm{F}$.

Les adultes sont parfois nombreux sur le tronc de Mahogany au cours de fortes infestations; ils courent par temps ensoleillé autour des galeries d'entrée afin d'y pondre et de capturer des adultes de Scolytes qu'ils consomment. Les larves sont dans les galeries et se nourrissent de larves d'Hexacolus guyanensis. Ces observations concordent avec celles faites chez Enoclerus sphegeus, prédateur de Dendroctonus ponderosae (REID 1957, SCHMID 1970) ainsi que sur Thanasimus undatulus (AMmAN 1972).

Au cours de l'étude de l'évolution de la population de Scolytes pendant la dégradation du Mahogany, nous avons noté l'importance des larves de Cléride dans les galeries (fig. 7). Elles apparaissent dès le début de l'attaque, ce qui semble confirmer l'observation de CAMORS et PAYNe (1973) : Thanasimus dubius arrive en grand nombre dès le début des attaques de Dendroctonus frontalis.

En éclosoir (fig. 8), un nombre important de ce prédateur est sorti en même temps que les générations $\mathrm{G} 1$ et $\mathrm{g} 1$ de Scolytes. Ne retournant pas pondre dans le même tronc, l'action de $M$. pici ne s'est plus fait sentir au cours des générations suivantes de Scolytes, dont les maxima ont été beaucoup plus importants.

\section{d. Acariens et Nématodes}

Il est commun de rencontrer des Acariens dans les galeries, mais ils sont généralement saprophages. Par contre, certains sont fixés en grand nombre sur la partie ventrale des adultes.

Un Nematode Sphaerulariidae a également été récolté à l'intérieur du corps des Scolytes. Les dimensions et la fécondité des femelles permettent de conclure quant à son rôle de parasite vrai. Sa détermination, son cycle et son incidence sur les populations de Scolytes feront l'objet d'un autre travail.

\section{III. - IMPORTANCE DES ATTAQUES ET MODALITÉS D'INTERVENTIONS}

\section{1. - Évaluation des dégâts en 1969}

Les dégâts sont constatés dans les plantations lorsque les foyers sont déjà étcndus avec de nombreux arbres morts. De tels dégâts ont ainsi été découverts entre 1967 et 1969 , et le tableau 2 récapitule les pertes dans les diverses plantations.

Des attaques sont constatées dans d'anciennes pépinières abandonnées, les arbres étant serrés de façon excessive $(0,5 \times 0,5 \mathrm{~m})$ comme dans des plantations normalement entretenues (écartement de l'ordre de 2 à 3 mètres), mais l'absence de plantations très âgées empêche de conclure quant à l'importance de ce facteur sur celle des attaques. 


\section{2. - Coupes sanitaires et leur efficacité}

Dès les premiers foyers découverts nous avons préconisé d'effectuer la coupe suivic du brûlage de tous les arbres attaqués. Ceci était envisageable dans la mesure où la plupart des foyers étaient de dimensions relativement restreintes, et c'était le seul moyen certes brutal, mais qui nous permettait d'espérer de limiter la dispersion et la multiplication de ces foyers.

\section{TABLEAU 2}

Bilan des attaques de Scolytes sur Mahogany du Honduras (1967-1969)

Importance of the bark beetle infestations in the mahogany plantations during the years 1967 to 1969

\begin{tabular}{|c|c|c|c|c|c|}
\hline $\begin{array}{l}\text { Unité de } \\
\text { plantation }\end{array}$ & $\begin{array}{l}\text { Surface } \\
\text { totale } \\
\text { de l'unité }\end{array}$ & $\begin{array}{l}\text { Année de } \\
\text { plantation }\end{array}$ & $\begin{array}{l}\text { Surface } \\
\text { touchée }\end{array}$ & $\begin{array}{l}\text { Nombre } \\
\text { d'arbres } \\
\text { détruits }\end{array}$ & $\begin{array}{c}\text { Années des } \\
\text { dégâts }\end{array}$ \\
\hline $\begin{array}{l}\text { Desbonnes } \\
\text { Dos d'âne } \\
\text { Baillargent } \\
\text { Ricart } \\
\text { Ilet Layette } \\
\text { Latour Sauteuil } \\
\text { Duportail } \\
\text { Guyonneau } \\
\text { Marolles }\end{array}$ & $\begin{array}{l}180 \text { ha } \\
30 \text { ha } \\
32 \text { ha } \\
34 \text { ha } \\
105 \text { ha } \\
159 \text { ha } \\
75 \text { ha } \\
33 \text { ha }\end{array}$ & $\begin{array}{l}\text { Pépinière } 1949 \\
1954 \text { à } 1956 \\
1955 \text { à } 1956 \\
1954 \text { à } 1958 \\
1954 \text { et } 1958 \\
1955-1958 \\
1949\end{array}$ & $\begin{array}{l}5,5 \\
3,92 \\
2,1 \\
0,13\end{array}$ & $\begin{array}{r}225 \\
8800 \\
2749 \\
3300 \\
111 \\
395 \\
400 \\
508 \\
330 \\
850 \\
760 \\
1413 \\
35\end{array}$ & $\begin{array}{l}1967 \\
1968 \\
1969 \\
1968 \\
1969 \\
1967 \\
1967 \\
1968 \\
1967 \\
1967 \text { à } 1969 \\
1967 \text { à } 1969 \\
1967-1968 \\
1967\end{array}$ \\
\hline
\end{tabular}

13 à 17 ha ont ainsi été détruits sur un total de 650 ha de plantations pour les massifs touchés, ceci en 3 ans, sur des arbres ayant au moins 10 ans. La soudaineté de ces pullulations inconnues jusqu'alors et les surfaces touchées donnent une idée de l'ampleur du problème et des risques encourus par l'ensemble des plantations.

Pour vérifier l'efficacité de ces coupes sanitaires, nous avons surveillé plusieurs d'entre elles par des visites régulières avec comptage du nombre d'arbres attaqués.

Ainsi à Duportail, 14 petits foyers allant de 5 à 48 arbres ont subi ces coupes en juin 1970 totalisant 292 arbres. Les plantations datent de 1958 et la situation de ces foyers tant en pente raide que sur une crête ou dans un fond, du côté du vent comme du côté protégé du vent, ne nous permettent pas de définir des sites attractifs pour les insectes essaimant. Trois mois après la coupe, 40 arbres attaqués et sur pied, la plupart déjà morts, ont été comptés pour l'ensemble des foyers, mais les visites mensuelles ont montré 4 arbres nouvellement attaqués en 1 an. C'est dire que lors de la $1^{\text {re }}$ coupe, une quarantaine d'arbres à un stade précoce d'attaque avaient été laissés sur pied mais que le nombre insuffisant d'insectes n'a pas permis la création de nouveaux foyers.

Des observations analogues ont été faites dans les plantations de RiCARD. Six foyers ont subi une coupe sanitaire en avril 1971, complété d'une visite et de l'abattage des nou- 
veaux arbres attaqués 6 mois plus tard. La seconde coupe s'est avérée réduite à quelques arbres par foyer.

Dans l'un d'eux, les arbres coupés ont été laissés sur le sol sans être brûlés : les insectes essaimant ont attaqué à nouveau les arbres sur pied, à proximité de leur base. La plupart de ces attaques ont avorté; les insectes étant noyés par la sève. En général les arbres sont attaqués à une hauteur de l'ordre de 2 à 3 mètres du sol, sans doute en relation avec la pression osmotique de la sève, comme Chararas l'a démontré (1970).

\section{3. - Évolution naturelle des foyers}

Les coupes sanitaires en détruisant une forte proportion de la population d'insectes, n'en laissent pas suffisamment pour qu'une nouvelle pullulation puisse se produire. Il semble qu'un seuil critique existe, en deçà duquel l'attaque avorte, et au-delà duquel elle se développe, tant au niveau d'un arbre que d'un foyer.

Dans les plantations de Desbonnes, 5,5 ha ont été détruits par $H$. guyanensis et nettoyés par une coupe sanitaire en 1968, totalisant 8800 arbres. En 1969, 2750 arbres, près de 4 ha sont détruits dans le voisinage et également nettoyés. Nous observons cependant l'année suivante une pullulation touchant quelques centaines d'arbres, en bordure de la coupe de l'année précédente. En 1971, nous constatons un prolongement de cette pullulation et comptons le 5 mai 79 arbres touchés, dont 67 morts, 9 avec des feuilles sèches, 2 avec d'abondantes exsudations et 1 en début d'attaque. Par des visites de ce foyer pendant 7 mois, nous notons sa rapide extinction, avec aucune extension vers des arbres sains.

Ainsi le nettoyage d'un foyer étendu sur plusieurs hectares a laissé suffisamment d'arbres en début d'attaque par $H$. guyanensis sur pied pour que de nouvelles pullulations puissent se développer. La phase croissante de telles pullulations avec multiplication des insectes, et essaimage vers les arbres voisins semble rapide puisque en l'espace de quelques mois des dizaines, voir des centaines d'arbres peuvent être détruits. S'il ne nous a pas été possible de suivre de telles croissances de pullulation, par le fait que leur existence n'est constatée généralement qu'à un stade avancé, nous avons cherché à déterminer les facteurs intervenant dans leur déclin.

Il semblerait que les attaques soient déjà importantes au moment de la reprise des pluies, ce qui voudrait dire que le début des attaques correspondrait avec celui de la saison sèche, mais les observations faites à un stade d'évolution des populations déjà trop tardif et la très grande variabilité empêche de conclure sur ce point. De même les arbres changent de feuilles un peu toute l'année et l'action des facteurs climatiques sur cet aspect de la phénologie est inconnue.

Les figures 9 et 10 présentent l'évolution de 2 foyers étudiés pendant plus de 6 mois à Celus et dans la parcelle de Desbonnes 1949, avec comptage à chaque visite du nombre d'arbres atteints et notation de leur état.

Parallèlement, des échantillons de morceaux d'écorce furent prélevés et disséqués au laboratoire avec dénombrement des orifices de galeries, d'adultes et de larves d'H. guyanensis vivants et morts, de cas de mycose, des Clérides. La présence de Nématodes et d'acariens fut contrôlée. 


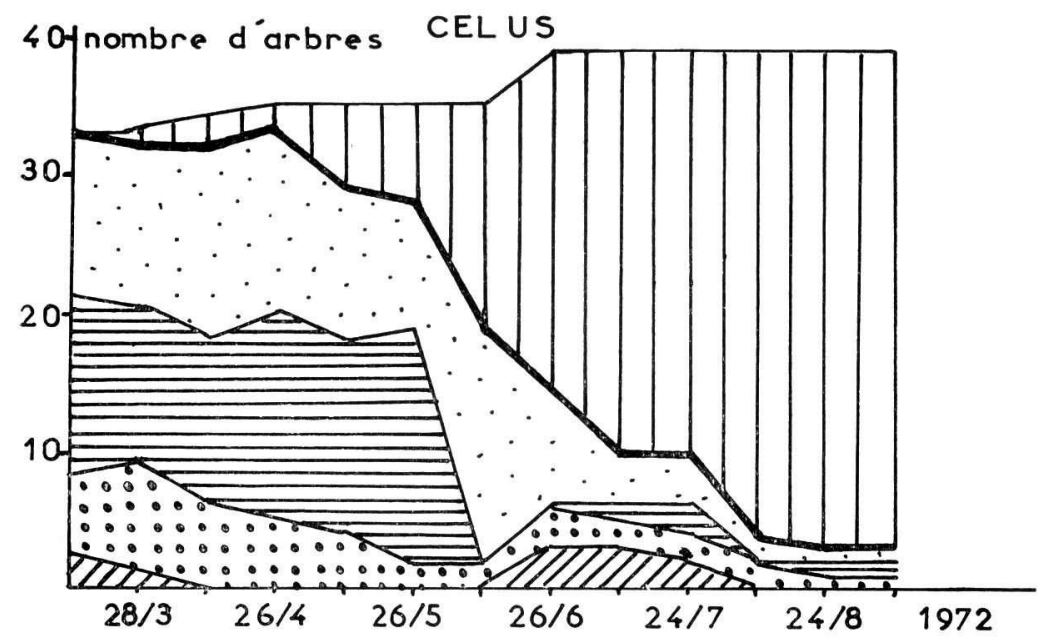

Fig. 9. - Évolution d'un foyer dans la parcelle Pépinière à Celus en 1972

FIG. 9. - Evolution of an infestation in an old nursery at Celus in 1972

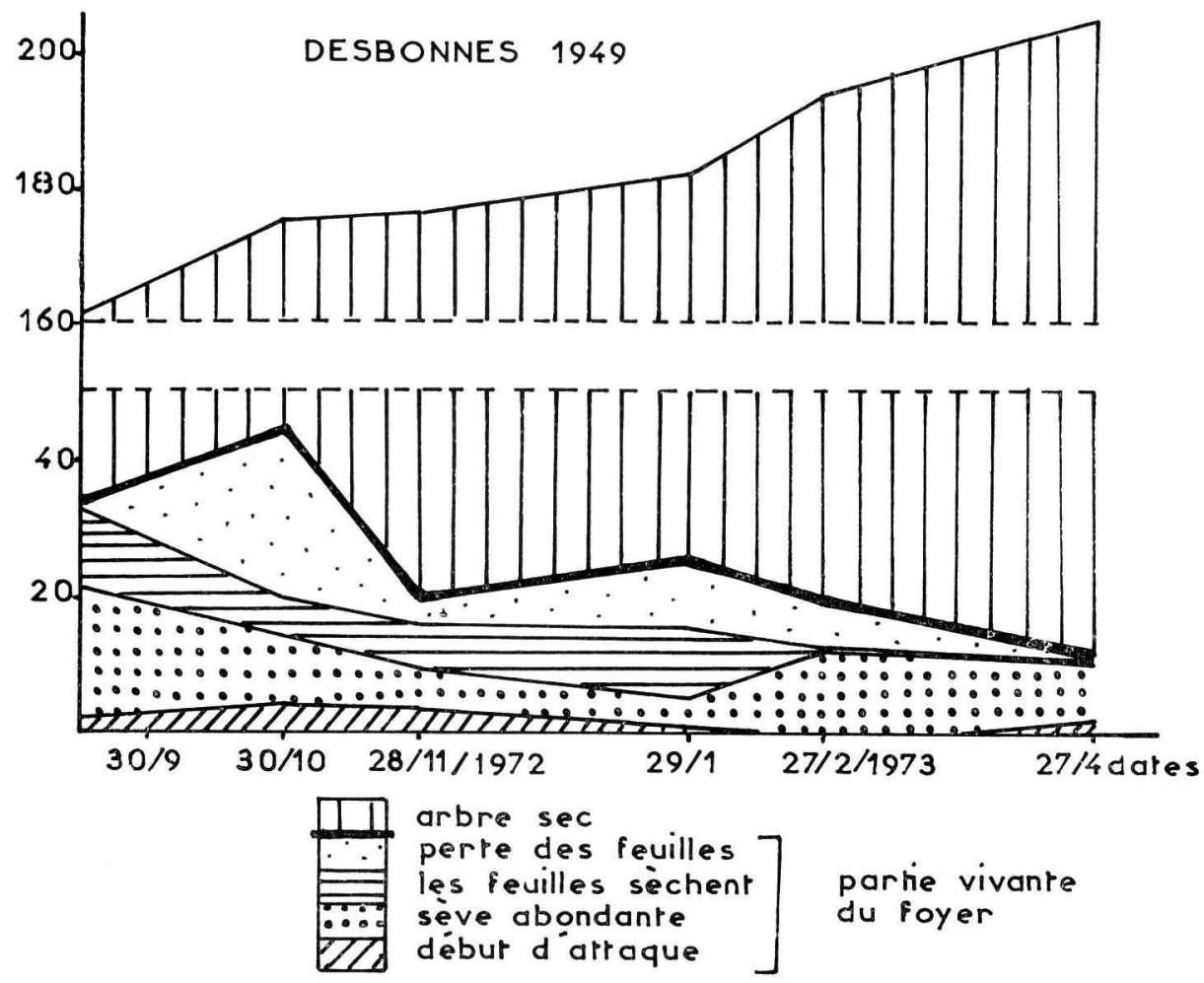

Fig. 10. - Évolution d'un foyer dans la parcelle Desbonnes 1949 en 1972-1973

FIG. 10. - Évolution of an infestation in Desbonnes 1949 patch, in 1972-1973 
Dans les 2 cas la phase active de la pullulation diminue : le nombre d'arbres contenant encore des insectes vivants, c'est-à-dire des arbres non déjà secs, décroît avec le temps.

Cette décroissance est rapide à Celus : le foyer s'est développé dans une ancienne pépinière abandonnée, où les arbres sont très serrés et chétifs, avec des troncs de quelques centimètres de diamètre malgré leur âge $(11$ ans). Des 150 arbres touchés, nous n'avons considéré que les survivants après notre première visite. La faible épaisseur du phloème ne permet pas à plusieurs générations de se développer, aussi le dépérissement de l'arbre est rapide.

A Desbonnes, de nouveaux arbres sains sont attaqués, mais l'ensemble de la pullulation diminue.

Nous avons tenté de caractériser dans les 2 sites les principales phases croissantes (pleine exsudation) et décroissante (feuilles jaunes et feuilles tombées) des attaques par la comparaison des populations vivantes et mortes d' $H$. guyanensis, ainsi que par l'importance de l'action des facteurs biotiques.

\section{TABLEAU 3}

Population d'H. guyanensis, de Cléride (Madoniella pici) et présence de la mycose à Metarrhizium anisopliae dans les Mahoganys attaqués de Desbonnes et de Celus Populations of $\mathbf{H}$. guyanensis, of the Clerid beetle (Madoniella pici) and importance of the green muscardine (Metarrhizium anisopliae) in the infested trees at Desbonnes and Celus

Nombre d'arbres échantillonnés

Surface d'écorce disséquée pour $100 \mathrm{~cm}^{2}$, nombre de :

Orifice de galeries

Hexacolus vivants

» morts / nombre

» / / pourcentage

Mycoses : nombre

en $\%$ des Hexacolus morts

Clérides

\begin{tabular}{|c|c|c|c|}
\hline \multicolumn{2}{|c|}{ Desbonnes } & \multicolumn{2}{|c|}{ Celus } \\
\hline $\begin{array}{c}\text { Pleine } \\
\text { exsudation }\end{array}$ & $\begin{array}{c}\text { Perte } \\
\text { des feuilles }\end{array}$ & $\begin{array}{c}\text { Pleine } \\
\text { exsudation }\end{array}$ & $\begin{array}{c}\text { Perte } \\
\text { des feuilles }\end{array}$ \\
\hline 30 & 15 & 6 & 7 \\
\hline $1200 \mathrm{~cm}^{2}$ & $600 \mathrm{~cm}^{2}$ & $240 \mathrm{~cm}^{2}$ & $280 \mathrm{~cm}^{2}$ \\
\hline 168 & 172 & 222 & 263 \\
\hline 147 & 84 & 92,1 & 32,1 \\
\hline 4,7 & 4,2 & 68,7 & 100,3 \\
\hline $3 \%$ & $11,5 \%$ & $42,7 \%$ & $75,7 \%$ \\
\hline 0,2 & 0,5 & 54,2 & 78,9 \\
\hline $3,6 \%$ & $12,0 \%$ & $78,8 \%$ & $78,6 \%$ \\
\hline 2 & 6,3 & 8,7 & 3,2 \\
\hline
\end{tabular}

Ce tableau suscite les remarques suivantes : la densité en insectes est supérieure au moment de la pleine exsudation, le nombre d'orifice de galerie l'étant plus tardivement, comme nous l'avons déjà mentionné (fig. 7). La proportion d'insectes morts augmente avec l'âge des attaques mais alors qu'à Desbonnes elle reste peu importante et que l'action des Clérides et de la mycose est réduite, elle est très forte à Celus et atteint $75 \%$ de la population d'insectes dans le tronc. Metarrhizium joue ici un rôle prépondérant puisque près de $80 \%$ des cadavres d'insectes portent des spores au moment des dissections. Les Clérides sont plus nombreux en début d'attaque, mais nous avons observé des cas de mycose sur Madoniella pici.

C'est au cours du mois de mai que le maximum d'arbres perdent leurs feuilles à Celus, 
et c'est à cette période que le nombre de mycoses est le plus important, correspondant à la reprise des pluies après la saison sèche. $M$. anisopliae est ici le facteur clé de mortalité des Scolytes, entraînant le déclin rapide de la pullulation.

\section{4. - La télédétection dans la recherche des foyers}

Au stade actuel de nos recherches, les coupes sanitaires constituent le moyen de protection le plus efficace. Cependant il est nécessaire d'assurer une surveillance des plantations afin de détecter les foyers à un stade aussi précoce que possible. Les éclaircies n'étant réalisées normalement que tous les 5 ans, et les plantations occupant la plupart du temps des terrains très accidentés, la visite des diverses parcelles constitue un travail long, fastidieux et obligatoirement incomplet.

Aussi nous avons profité du passage de la mission aéroportée « Ressources terrestres en Guadeloupe » du Centre National d'Études Spatiales en mars 1972 pour demander des prises de vues de diverses plantations dans lesquelles nous connaissions la présence de foyers de Scolytes. Trois émulsions ont été utilisées : noir et blanc panchromatique, couleur vraie et fausse couleur Infra rouge. Seul le peuplement de Celus a été couvert par les photographies. La comparaison des agrandissements de la zone intéressante dans les 3 émulsions est présentée dans le tableau 4.

\section{TABLEAU 4}

Comparaison des 3 émulsions utilisées en photographie aérienne au-dessus de la plantation de Celus Comparison of the 3 films used for aerial pictures of the Celus plantation

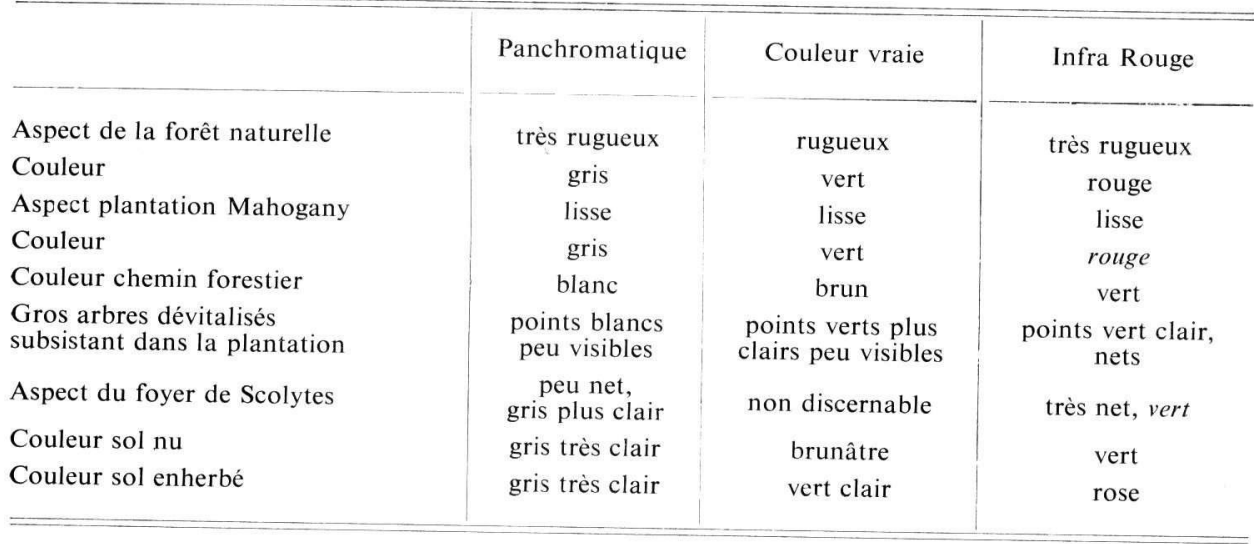

La photographie fausse couleur Infra rouge donne des résultats nettement supérieurs aux autres, les arbres morts apparaissent en vert sur fond rouge. Le pouvoir de résolution est suffisant pour distinguer aisément un foyer de quelques dizaines de mètres, alors que les prises de vues ont été effectuées à 3500 mètres d'altitude avec une caméra WILD. L'agrandissement photographique augmente encore le pouvoir de résolution qui peut atteindre $10 \mathrm{~cm}$ au sol pour des prises de vues réalisées à 2500 mètres d'altitude.

A proximité, une ancienne pullulation de Scolytes avait laissé environ un hectare d'arbres morts, la plupart coupés lors d'un nettoyage du foyer. Ces dégâts sont parfaitement visibles sur les photographies aériennes. 
Cette technique, présentée récemment par SCHNEIDER (1972), a déjà été utilisée pour détecter des dégâts d'insectes (HART et MYERs 1968). Les quelques résultats que nous avons obtenus sont suffisamment nets pour qu'on puisse préconiser ce mode de détection, chaque fois que des possibilités techniques s'offriront.

\section{CONCLUSIONS}

H. guyanensis est un Scolytini ravageur primaire de feuillus importé récemment en Guadeloupe. Les galeries maternelles sont transversales en accolade de part et d'autre de la chambre d'accouplement. Le cycle de développement dure 2 à 3 semaines et une partie des jeunes adultes reste dans les galeries maternelles pour s'y accoupler et pondre. 4 stades larvaires sont mis en évidence par mensuration des capsules céphaliques. Cette espèce semble inféodée à quelques représentants de la famille des Meliacées.

Seuls les arbres de plus de 10 ans sont attaqués. Les galeries des premiers insectes entraînent un écoulement de sève qui participe à l'attraction en masse d'autres Scolytes. La pullulation est alors rapide avec développement dans le phloème d'un grand nombre de larves, et les générations se suivent jusqu'à consommation totale du liber. L'arbre est totalement sec. Quelques autres Scolytes se rencontrent parfois dans l'acajou, ainsi que diverses mycoses dont l'agent de la Muscardine verte, Metarrhizium anisopliae ainsi que le Cléride Madoniella pici dont nous avons décrit la larve.

D'importants foyers d'attaque furent découverts dans les plantations à partir de 1967, avec perte en 3 ans de plus de $2 \%$ des arbres dans les massifs touchés. Les coupes sanitaires préconisées, avec brûlage sur place des arbres attaqués abattus se sont avérées très efficaces malgré leur coût élevé dû à l'emploi d'une main-d'œuvre nombreuse. Une visite des foyers 3 mois après la coupe est une sécurité, surtout lorsque les attaques ont été importantes, car nous n'avons observé de nouvelle pullulation que lorsqu'un nombre suffisant d'arbres en début d'infestation a été laissé sur pied. Nous n'avons pu préciser les facteurs favorisant les pullulations, tels la situation des plantations attaquées sur une pente ou dans un fond, ou encore la période d'infestation au cours de l'année. L'alternance saison sèche-saison humide qui règle nombre de phénomènes biologiques dans le milieu tropical ne paraît pas conditionner les attaques. Par contre la reprise des pluies a nettement favorisé le développement d'une épizootie de la muscardine verte dans les populations de Scolytes à Celus, entraînant une extinction rapide de ce foyer. L'action des Clérides prédateurs n'est pas négligeable, mais $M$. anisopliae s'est trouvé être le facteur clé dans ce biotope alors que dans d'autres son action était moindre.

L'utilisation de techniques de télédétection pour le repérage des foyers a montré leur intérêt dans la protection des forêts.

Reçu pour publication en Mars 1974.

\section{SUMMARY}

BIOLOGY AND DAMMAGES OF HEXACOLUS GUYANENSIS SCHELD. IN MAHOGANY PLANTATIONS (Swietenia macrophylla KING. MELIACEE) IN GUADELOUPE

The biology of a primary pest of Mahogany tree, Hexacolus guyanensis, is studied in the laboratory and in the field. This species is monogamous and has 4 larval instars developing in 2 to 3 weeks in the liber. It seems to be infecffed to some species of the family of Meliacees. 
The evolution of beetle population during the degradation of the tree, the presence of associated fauna and desease are studied. The larvae of the Clerid beetle Madoniella pici is described.

With the importance of the dammages since 1967 in Guadeloupe, we recommand to cut and burn the infested trees. The efficiency of this method is verified. The development of two pullulations in mahogany plantations are studied in reference of the state of the trees, the populations of beetles with their parasites and desease. In one of them, the fungus Metarrhizium anisophiae is the main factor of reduction of the population.

The research of attacked areas in the plantations by teledetection proved the interest of the employing of infrared color films in comparison with the others.

\section{RÉFÉRENCES BIBLIOGRAPHIQUES}

Amman G. D., 1972. - Prey consumption and development of Thanasimus undatulus, a predator of the mountain pine beetle. Environ. Entomol., 1 (4), 528.

Asenjo C. F., Marin L. A., Torres W., Campillo A., 1958. - Termite repellent activity and chemical composition of West Indian mahogany wood, (Swietenia mahagoni Jacq.) with special reference to the P2 fraction. J. Agr. Un. Puerto-Rico XLII (3).

Bakke A., 1973. - Bark Beetle Pheromones and their Potential Use in Forestry. OEPP / EPPO Bull., (9) $5-15$.

Balachowsky A. S., 1963. - Famille des Scolytidae. Dans : Entomologie appliquée à l'Agriculture, Traité de Balachowsky, t. 1, vol. II, 1237-1289. Masson et Cie Éd. Paris.

Balogun R. A. 1970. - The life history and habits of the earch bark beetle Ips cembrae (col. Scolytidae) in the north east of Scotland. Canad ent., 10 (2) 226-239.

Berryman A. A., Ashraf M., 1970. - Effects of Abies grandis resin on the atack behavior and brood survival of Scolytus ventralis (Col. Scolytidae). Canad ent., 102 (10), 1229-1236.

Borden J. H., 1969. - Observations on the life history and habits of Alniphagus aspericollis (Col. Scolytidae) in southwestern british columbia. Can. Ent., 101, 870-878.

Camors F. B., PAYNe T. L., 1973. - Sequence of arrival of Entomophagous Insects to trees infested with the Southern Pine beetle. Envir. Entom., 2 (2), 267-270.

Chararas C., 1970. - Écologie des Scolytidae. Bull. Soc. Écol., 3, 169-188.

Fleutiaux E., Legros C., Lepesne P., Paulian R., 1947. - Faune de l'empire français. Coléoptère des Antilles françaises. Office de la Rech. Sc. Coloniale. 239.

Hart W. G., Myers V. I., 1968. - Infrared aerial color photography for detection of populations of Brown soft scale in Citrus groves. J. Ec. Ent., 61 (3), 617-725.

Lamb B., 1966. - Mahogany of tropical america. Press of the University of Michigan, 220.

Lintle E. L., Wadsworth F. H. - Common trees of Puerto Rico and the Virgin Island. Agric. Handbook $n^{\circ}$ 249, USDA, Washington, 548.

MILNE D. M., 1970. - Interpretation of population data for the Columbian timber beetle Corthylus columbianus HOFKINS (Col. Scolytidae) derived from naturally preserved indices. Diss. Abstr. B., $30(12), 5542-\mathrm{B}$.

Moore G. C., 1970. - Isolating entomogenous fungi and Bacteria, and tests of fungal isolates against the southern pine beetle. J. Ec. Ent., 63 (5), 1702-1703.

Pesson P., Chararas C., 1969. - Les Scolytes, insectes ravageurs mondiaux des forêts de conifères. Ann. Biol., 8 (11-12), 683-733.

REID R. W., 1957. - The bark beetle complex associated with lodgepole pine slash in Alberta. Part III Notes on the biologies of several predators with special reference to Enoclerus sphegeus Fab. (Coleoptera : Cleridae) and two species of mites. The canadian Entomol., 89, 111-120.

Renwick J. A. A., ViTe J. P., 1969. - Bark beetle attractants : mecanism of colonization by Dendroctonus frontalis. Nature, 224 (5225), 1222-1223.

Renwick J. A. A., Vite J. P., 1970. - Systems of chemical communication in Dendroctonus. Contrib. Boyn Thompson Inst., 24, 283-292.

SснміD J. M., 1970. - Enoclerus sphegeus (Col. Cleridae), a predator of Dendroctonus ponderosae (Col. Scolytidae) in the blark hills. Canad. ent., $102(8), 969-977$.

SCHNeIDER S., 1972. - Methodes modernes de reconnaissance par la photographie aérienne mises au service des recherches sur l'environnement. Photo. Technik und Wietschaft., 4, 92-96.

WATSON J. A., 1970. - Rhythmic emergence patterns of the mountain pine beetle. Dendroctonus ponderosae. (Col. Scolytidae). Can. Ent., 102, 1054-1056. 